\title{
THE OPEN-OPEN TOPOLOGY FOR FUNCTION SPACES
}

\section{KATHRYN F. PORTER}

\author{
Department of Mathematical Sciences \\ Saint Mary's College of California \\ Moraga, CA. 94575
}

(Recefved February 20, 1991 and in revised form January 22, 1992)

\begin{abstract}
Let $(X, T)$ and $\left(Y, T^{*}\right)$ be topological spaces and let $F \subset Y^{X}$. For each $U \in T, V \in$ $T^{*}$, let $(U, V)=\{f \in F: f(U) \subset V\}$. Define the set $S_{o o}=\left\{(U, V): U \in T\right.$ and $\left.V \in T^{*}\right\}$. Then $S_{o o}$ is a subbasis for a topology, $T_{o o}$ on $F$, which is called the open-open topology. We compare $T_{o o}$ with other topologies and discuss its properties. We also show that $T_{o o}$, on $H(X)$, the collection of all self-homeomorphisms on $X$, is equivalent to the topology induced on $H(X)$ by the Pervin quasi-uniformity on $X$.
\end{abstract}

KEY WORDS AND PHRASES. Compact-open topology, admissible topology, Galois space, Pervin quasi-uniformity, self-homeomorphism, quasi-uniform convergence.

1980 AMS SUBJECT CLASSIFICATION CODES. Primary 54C35, Secondary 54H13.

\section{INTRODUCTION.}

The type of set-set topology which will be discussed here is one which can be defined as follows: Let $(X, T)$ and $\left(Y, T^{*}\right)$ be topological spaces. Let $\mathbf{U}$ and $\mathbf{V}$ be collections of subsets of $X$ and $Y$, respectively. Let $F \subset Y^{X}$, the collection of all functions from $X$ into $Y$. Define, for $U \in \mathbf{U}$ and $V \in \mathbf{V},(U, V)=\{f \in F: f(U) \subset V\}$. Let $S(\mathbf{U}, \mathbf{V})=\{(U, V): U \in \mathbf{U}$ and $V \in \mathbf{V}\}$. If $S(\mathbf{U}, \mathbf{V})$ is a subbasis for a topology $T(\mathbf{U}, \mathbf{V})$ on $F$ then $T(\mathbf{U}, \mathbf{V})$ is called a set-set topology.

One of the original set-set topologies is the compact-open topology, $T_{c o}$, which was introduced in 1945 by R. Fox [1]. For this topology, as one may surmise from the name, $U$ is the collection of all compact subsets of $X$ and $\mathbf{V}=T^{*}$, the collection of all open subsets of $Y$. Fox and Arens [2] developed and examined the properties of this now well-known topology. In particular, it was shown that if $F \subset C(X, Y)$, the collection of all continuous functions on $X$ into $Y$, then $T_{c o}$ on $F$ is equivalent to the topology of uniform convergence on compacta; and, if in addition, $X$ is compact, then $T_{c o}$ is equivalent to the topology of uniform convergence on $F$. Arens also defined 
the concept of admissible topology for function spaces and was instrumental in the study of groups of self-homeomorphisms and topological groups.

Other set-set topologies that have been of interest are: the point-open topology, $T_{p}$, also known as the topology of pointwise convergence, in which $\mathbf{U}$ is the collection of all singletons in $X$ and $\mathrm{V}=T^{*}$; the closed-open topology, where $\mathrm{U}$ is the collection of all closed subsets of $X$ and the set $\mathbf{V}=T^{*} ;$ and the bounded-open topology (Lambrinos [3]), where $\mathbf{U}$ is the collection of all bounded subsets of $X$ and again, $\mathrm{V}=T^{*}$.

In section 2 of this paper, we shall introduce and discuss the open-open topology, $T_{o o}$, for function spaces. It will be shown which of the desirable properties $T_{o o}$ possesses. In section 3 , the group of all self-homeomorphisms, $H(X)$, endowed with $T_{o o}$, is discussed.

As will be proven in section $5, T_{o o}$, on $H(X)$, is actually equivalent to the Pervin topology of quasi-uniform convergence (Fletcher [4]). One of the advantages of the open-open topology is the set-set notation which provides us with simple notation and, hence, our proofs are more concise than those using the cumbersome notation of the quasi-uniformity. Pervin spaces will be discussed in section 4 .

We assume a basic knowledge of quasi-uniform spaces. An introduction to quasi-uniform spaces may be found in Fletcher and Lindgren's [5] or in Murdeshwar and Naimpally's book [6].

Throughout this paper we shall assume $(X, T)$ and $\left(Y, T^{*}\right)$ are topological spaces.

\section{THE OPEN-OPEN TOPOLOGY.}

If we let $\mathbf{U}=T$ and $\mathbf{V}=T^{*}$, then $S_{o o}=S(\mathbf{U}, \mathbf{V})$ is the subbasis for the topology, $T_{o o}$, on any $F \subset Y^{X}$, which is called the open-open topology.

We first examine some of the properties of function spaces the open-open topology possesses.

THEOREM 1. Let $F \subset C(X, Y)$. If $\left(Y, T^{*}\right)$ is $\mathrm{T}_{\mathrm{i}}$ for $\mathrm{i}=0,1,2$, then $\left(F, T_{o o}\right)$ is $\mathrm{T}_{\mathrm{i}}$ for $\mathrm{i}$ $=0,1,2$.

PROOF. We shall show the case $\mathrm{i}=2$; the other cases are done similarly. Let $\mathrm{i}=2$. Let $f, g \in F$ such that $f \neq g$. Then there is some $x \in X$ such that $f(x) \neq g(x)$. If $Y$ is $\mathrm{T}_{2}$ there exists disjoint open sets $O$ and $U$ in $Y$ such that $f(x) \in U$ and $g(x) \in O$. Both $f$ and $g$ are continuous, so there are open sets $V$ and $W$ in $X$ with $x \in V \cap W, f(V) \subset U$ and $g(W) \subset O$. $f \in(V, U), g \in(W, O)$, and $(V, U) \cap(W, O)=\phi$. Thus, $\left(F, T_{o o}\right)$ is $\mathrm{T}_{2}$.

A topology, $T^{\prime}$, on $F \subset Y^{X}$ is called an admissible (Arens [2]) topology for $F$ provided the evaluation map, E: $\left(F, T^{\prime}\right) \times(X, T) \rightarrow\left(Y, T^{*}\right)$, defined by $E(f, x)=f(x)$, is continuous.

THEOREM 2. If $F \subset C(X, Y)$ then $T_{o o}$ is admissible for $F$.

PROOF. Let $F \subset C(X, Y)$. Let $O \in T^{*}$ and let $(f, p) \in E^{-1}(O)$. Then $f(p) \in O$. Since $f$ is continuous, there exists some $U \in T$ such that $p \in U$ and $f(U) \subset O$. So, $(f, p) \in(U, O) \times U$. If $(g, b) \in(U, O) \times U$, then $g(U) \subset O$ and $b \in U$, so $g(b) \in O$. Hence, $(U, O) \times U \subset E^{-1}(O)$. Therefore, $T_{o o}$ is admissible for $F$. 
Arens also has shown that if $T^{\prime}$ is admissible for $F \subset C(X, Y)$, then $T^{\prime}$ is finer than $T_{c o}$. From this fact and Theorem 2, it follows that $T_{\text {co }} \subset T_{o o}$.

\section{THE OPEN-OPEN TOPOLOGY ON $H(X)$.}

We now consider $T_{o o}$ on $H(X)$, the collection of all self-homeomorphisms on $X$. Note that $H(X)$ with the binary operation $\circ$, composition of functions, and identity element $e$, is a group.

Some of the set-set topologies previously mentioned are equivalent under certain hypotheses. For example, the closed-open topology is equal to the compact-open topology whenever $X$ is compact $T_{2}$, the point-open topology is equivalent to the compact-open topology if all compact subsets of $X$ are finite sets. It is always advantageous to know when topologies are or are not equivalent. In particular, it is well known that if $X$ is $T_{1}$ then $T_{p} \subset T_{c o}$ and as we have shown $T_{c o} \subset T_{o o}$. When are $T_{c o}$ and $T_{o o}$ distinct? One hypothesis under which these two topologies are not equivalent is: "Let $X$ be $T_{2}$ and Galois."

A topological space is Galois provided that for each closed set, $C \subset X$ and each point $p^{\prime} \in X \backslash C$, there is an $h \in H(X)$ such that $h(x)=x$ for all $x \in C$ and $h(p) \neq p$. Among the spaces which are $T_{2}$ Galois are the topological vector spaces and, as Fletcher [7] has shown, locally euclidean $\mathrm{T}_{2}$ spaces or homogeneous 0-dimensional spaces which have no isolated points.

THEOREM 3. If $X$ is a $T_{2}$ Galois space then $T_{o o} \neq T_{c o}$ on $H(X)$.

PROOF. Let $X$ be a $T_{2}$ Galois space. Let $x \in X$; then $X \backslash\{x\}$ is open in $X$ so that $(X \backslash\{x\}, X \backslash\{x\})$ is open in $T_{o o}$. But note that $(X \backslash\{x\}, X \backslash\{x\})=(\{x\},\{x\})$ in $\left(H(X), T_{o o}\right)$.

Let $e$ be the identity map on $X$ then $e \in(\{x\},\{x\})$. Claim: $(\{x\},\{x\}) \notin T_{c o}$ and hence $T_{\text {co }} \neq T_{o o}$. Let $\bigcap_{i=1}^{n}\left(C_{i}, U_{\imath}\right)$ be a basic open set in $T_{\text {co }}$ which contains $e$. So, $C_{i} \subset U_{i}$ for all $i=1,2,3, \ldots, n$.

Set $U_{0}=X$ and $C_{0}=\phi$. Let $P=\left\{U_{i}\right\}_{i=0}^{n}$ and $Q=\left\{C_{i}\right\}_{i=0}^{n}$. Define $P_{x}=\cap\{U \in P \mid x \in U\}$ and $Q_{x}=\cup\{C \in Q \mid x \notin C\}$. Let $L=P_{x} \backslash Q_{x}$. Note that $x \in L$ and $L$ is open in $X$.

Case 1: $L=X:$ Then for each $i=1,2,3 \ldots, n, U_{\imath}=X$, so that $\bigcap_{i=1}^{n}\left(C_{\imath}, U_{\imath}\right)=H(X)$ and $\bigcap_{i=1}^{n}\left(C_{i}, U_{i}\right) \not \subset(\{x\},\{x\})$.

Case 2: $L \neq X$ : Thus, since $X$ is Galois, there exists some $h \in H(X)$ such that $h(y)=y$ for all $y \in X \backslash L$ and $h(x) \neq x$. So $h \notin(\{x\},\{x\})$ and $h \in(L, L)$. Let $q \in C$, for some $j \in\{1,2, \ldots, n\}$. If $q \notin L$ then $h(q)=q \in C_{\mathrm{j}} \subset U_{\mathrm{j}}$. If $q \in L$ then $x \in C_{j}$ from which it follows that $L \subset U_{j}$. Thus, $h(L)=L \subset U_{\jmath}$. In either case, $h \in \bigcap_{i=1}^{n}\left(C_{t}, U_{\imath}\right)$ and again $\bigcap_{\imath=1}^{n}\left(C_{\imath}, U_{\imath}\right) \not \subset(\{x\},\{x\})$.

Therefore, $(\{x\},\{x\}) \notin T_{c o}$ and $T_{c o} \neq T_{00}$.

Effros' Theorem (Effros [8]) is a widely known and useful tool in the study of homogeneous spaces and continua theory. Of its several forms, the most popular is: If $X$ is a compact homogeneous metric space then for each $x \in X$, the evaluation map, $E_{x}:\left(H(X), T_{c o}\right) \rightarrow X$, defined by $E_{x}(h)=h(x)$, is an open map. It follows that, if the conclusion holds when $E_{x}$ is considered on the 
space $(H(X), T *)$, and if $T \subset T *$ on $H(X)$, then the conclusion also holds on $(H(X), T)$. Ancel [9] has asked the following question: If the hypothesis of the Effros' Theorem is changed to " $X$ is a compact, homogeneous, Hausdorff space,"is the evaluation map on $\left(H(X), T_{c o}\right)$ still open *? To this end, since $T_{c o} \subset T_{o o}$, we could consider whether a form of Effros' Theorem would be true for $T_{o o}$ on $H(X)$. Unfortunately, we discover the following.

THEOREM 4. Let $(X, T)$ be a $T_{t}$ topological space. Then, for each $x \in X$, the evaluation map, $E_{x}:\left(H(X), T_{o o}\right) \rightarrow X$ defined by $E_{x}(h)=h(x)$, is open only if $T$ is the discrete topology.

PROOF. Let $x \in X$. Then the set $O=((X \backslash\{x\}),(X \backslash\{x\}))$ is open in $\left(H(X), T_{o o}\right)$. But $((X \backslash\{x\}),(X \backslash\{x\}))=(\{x\},\{x\})$. So. $E_{x}(O)=\{x\}$. Thus $E_{x}$ is open for each $x \in X$ only if $X$ is discrete.

Let $(G, \circ)$ be a group such that $(G, T)$ is a topological space, then $(G, T)$ is a topological group provided the following two maps are continuous. (1) $m: G \times G \rightarrow G$ defined by $m\left(g_{1}, g_{2}\right)=g_{1} \circ g_{2}$ and $\Phi: G \rightarrow G$ defined by $\Phi(g)=g^{-1}$. If only the first map is continuous, then we call $(G, T)$ a quasi-topological group (Murdeshwar and Naimpally [6]).

It is not difficult to show that if $(X, T)$ is a topological space and $G$ is a subgroup of $H(X)$ then $\left(G, T_{o o}\right)$ is a quasi-topological group. However, $\left(G, T_{o o}\right)$ is not always a topological group as the following example (Fletcher [4]) shows: Let $X=R$ and let the topology on $X$ be described as follows: $T=\{(a, b) \subset R: a<0<b\} \cup\{\phi, X\}$. Let $f, g: X \rightarrow X$ be defined by $f(x)=-x$ and $g(x)=-\frac{x}{2}$. Clearly, $f$ and $g$ are homeomorphisms on $X$. Note that $f(x)=f^{-1}(x)$ and $g^{-1}(x)=-2 x$. Let $U=V=(-1,1)$. Then $f \in(U, V) \in T_{\text {oo }}$. Now define $\Phi: H(X) \rightarrow H(X)$ by $\Phi(h)=h^{-1}$. So, $f \in \Phi^{-1}((U, V))$. Claim: $\Phi$ is not continuous: Let $O=\bigcap_{i=1}^{n}\left(\left(a_{\imath}, b_{\imath}\right),\left(c_{i}, d_{\imath}\right)\right)$ be a basic open set in $\left(H(X), T_{o o}\right)$ which contains $f$. Then $a_{i}<0<b_{i}$ and $c_{i}<0<d_{i}$ for each $i$. If $x \in\left(a_{i}, b_{i}\right)$ then $f(x)=-x \in\left(c_{i}, d_{i}\right)$. So, $g(x)=-\frac{x}{2} \in\left(c_{i}, d_{i}\right)$ and, hence, $g \in O$. Thus, every basic open set containing $f$ also contains $g$. But $g^{-1}(U) \not \subset V$ and so $g \notin \Phi^{-1}((U, V))$. Therefore, any basic open set containing $f$ is not contained in $\Phi^{-1}((U, V))$. Thus, $\Phi$ is not continuous and $\left(G, T_{o o}\right)$ is not a topological group.

\section{PERVIN SPACES.}

A topological space, $(X, T)$, is called a Pervin space (Fletcher [4]) provided that for each finite collection, $\mathcal{A}$, of open sets in $X$, there exists some $h \in H(X)$ such that $h \neq e$ and $h(U) \subset U$ for all $U \in \mathcal{A}$.

Topologies are rarely interesting if they are the trivial or discrete topology. To this end, we have:

THEOREM 5. $\left(H(X), T_{o o}\right)$ is not discrete if and only if $(X, T)$ is a Pervin space.

PROOF. First, assume that $(X, T)$ is a Pervin space. Let $W$ be a basic open set in $T_{o o}$

*This was recently answered in the negative by Bellamy and Porter [10]. 
which contains $e$; i.e. $W=\bigcap_{\imath=1}^{n}\left(O_{\imath}, U_{\imath}\right)$ where $O_{\imath} \subset U_{\imath}$ for each $i=1,2,3, \ldots, n$ and $O_{\imath}$ and $U_{\imath}$ are open in $X .\left\{O_{\imath}: i=1,2,3, \ldots, n\right\}$ is a finite collection of open sets in $X$, and $X$ is a Pervin space, hence, there exists some $h \in H(X)$ such that $h \neq e$ and $h\left(O_{\imath}\right) \subset O_{\imath} \subset U_{\mathfrak{\imath}}$. So, $h \in W$ and $h \neq e$. Since $\left(H(X), T_{o o}\right)$ is a quasi-topological group, $\left(H(X), T_{o o}\right)$ is not a discrete space.

Now assume that $\left(H(X), T_{o o}\right)$ is not discrete. Let $V$ be a finite collection of open sets in $X$. Let $O=\bigcap_{U \in V}(U, U)$. Then, $O$ is a basic open set in $\left(H(X), T_{o o}\right)$ which is not a discrete space. Hence, there exists $h \in O$ with $h \neq e$. So, $(X, T)$ is Pervin.

Fletcher [4] proved that the Pervin topology of quasi-uniform convergence on $H(X)$ is not discrete if and only if $(X, T)$ is Pervin. In order to prove this, Fletcher had to first introduce numerous definitions along with some mind boggling notation. The above proof, along with the few needed definitions involving $T_{o o}$, is an example of the simplification that the definition of $T_{o o}$ offers over the quasi-uniform definition and notation.

\section{THE PERVIN TOPOLOGY OF QUASI-UNIFORM CONVERGENCE.}

Recall that if $Q$ is a quasi-uniformity on $X$, then the topology, $T_{Q}$, on $X$, which has as its neighborhood base at $x, B_{x}=\{U[x]: U \in Q\}$, is called the topology induced by $Q$. The ordered triple $\left(X, Q, T_{Q}\right)$ is called a quasi-uniform space. A topological space, $(X, T)$ is quasi-uniformizable provided there exists a quasi-uniformity, $Q$, such that $T_{Q}=T$. In 1962, Pervin [11] proved that every topological space is quasi-uniformizable by giving the following construction.

Let $(X, T)$ be a topological space. For each $O \in T$, define the set $S_{O}=(O \times O) \cup((X \backslash O) \times X)$. Let $S=\left\{S_{O}: O \in T\right\}$. Then $S$ is a subbasis for a quasi-uniformity, $P$, for $X$, called the Pervin quasi-uniformity and, as is easily shown, $T_{P}=T$.

If $(X, Q)$ is a quasi-uniform space then $Q$ induces a topology on $H(X)$ called the topology of quasi-uniform convergence w.r.t $Q$, as follows: For each set $U \in Q$, let us define $W(U)=\{(f, g) \in$ $H(X) \times H(X):(f(x), g(x)) \in U$ for all $x \in X\}$. Then, $B(Q)=\{W(U): U \in Q\}$ is a basis for $Q^{*}$, the quasi-uniformity of quasi-uniform convergence w.r.t. $Q$ (Naimpally [12]). Let $T_{Q}$ - denote the topology on $H(X)$ induced by $Q^{*}$. $T_{Q^{*}}$ is called the topology of quasi-uniform convergence w.r.t. $Q^{*}$. If $P$ is the Pervin quasi-uniformity on $X T_{P^{*}}$ is the Pervin topology of quasi-uniform convergence.

At this time one could, once again, prove that $T_{P^{*}}$ is not discrete if and only if $(X, T)$ is a Pervin space, this time using the quasi-uniform structure [4]. We leave this to the reader.

We now show that the open-open topology is equivalent to the Pervin topology of quasiuniform convergence.

THEOREM 6. Let $(X, T)$ be a topological space and let $G$ be a subgroup of $H(X)$. Then, $T_{o o}=T_{P^{*}}$ on $G$.

PROOF. Let $(O, U)$ be a subbasic open set in $T_{\text {oo }}$ and let $f \in(O, U)$. Then $f(O) \subset U$. So $f \in W\left(S_{U}\right)[f]$. Hence, if $g \in W\left(S_{U}\right)[f],\left((f(x), g(x)) \in S_{U}\right.$ for all $x \in X$. If $x \in O, f(x) \in U$, 
which implies that $g(x) \in U$. Thus, $g \in(O, U)$ and $W\left(S_{U}\right) \subset(O, U)$. Therefore, $T_{o o} \subset T_{P}$.

Now let $V \in T_{P}$. and let $f \in V$. Then there exists $U \in P$ such that $f \in W(U)[f] \subset V$. Since $U \in P$, there is some finite collection, $\left\{U_{\imath}: i=1,2, \ldots, n\right\} \subset T$ such that $\bigcap_{i=1}^{n} S_{U_{1}} \subset U$. Define $A=\bigcap_{i=1}^{n}\left(f^{-1}\left(U_{i}\right), U_{i}\right) . A$ is an open set in $T_{o o}$. and $f \in A$. Assume $g \in A$ and let $x \in X$. If $f(x) \in U$, for some $j \in\{1,2, \ldots, n\}$, then $x \in f^{-1}\left(U_{\jmath}\right)$. So, $g(x) \in U_{\jmath}$, hence, $(f(x), g(x)) \in U, \times U, \subset S_{U}$. If $f(x) \notin U$, for some $j \in\{1,2, \ldots, n\}$ then $(f(x), g(x)) \in\left(X \backslash U_{j}, X\right) \subset S_{U}$, Hence, $(f(x), g(x)) \in$ $\bigcap_{i=1}^{n} S_{U_{1}} \subset U$, and it follows that $g \in W(U)[f] \subset V$ and $A \subset V$. So, $T_{o o}=T_{P}$.

ACKNOWLEDGEMENT. The author would like to thank the Committee for Faculty and Curriculum Development at Saint Mary's College of California for their financial support.

\section{REFERENCES}

1. FOX, R., On Topologies for Function Spaces, Bull. Amer. Math. Soc., 51 (1945), 429-432.

2. ARENS, R., Topologies for Homeomorphism Groups, Amer. J. Math., $\underline{68}$ (1946), 593-610.

3. LAMBRINOS, P., The Bounded-Open Topology on Function Spaces, Man. Math., $\underline{35}$ (1982) no. 1, 47-66.

4. FLETCHER, P., Homeomorphism Groups with the Topology of Quasi-uniform Convergence, Arch. Math., 22 (1971), 88-92.

5. FLETCHER, P. and LINDGREN, W., Quasi-uniform Spaces, Lecture Notes in Pure and Applied Mathematics, 77, Marcel Dekker, 1982.

6. MURDESHWAR, M., and NAIMPALLY, S., Quasi-Uniform Topological Spaces, Noordoff, 1966.

7. FLETCHER, P. and SNIDER, P., Topological Galois Spaces, Fund. Math., $\underline{68}$ (1970), 143-148.

8. EFFROS, E., Transformation Groups and $C^{*}$-Algebras, Ann. of Math., (2) 81 (1965), 38-55.

9. ANCEL, R., An Alternative Proof and Applications of a Theorem of E. G. Effros, Mich. Math. J., $\underline{34}$ (1987) no. $1,39-55$.

10. BELlamY, D., AND PORTER, K., A Homogeneous Continuum Which is Non-Effros, Proc. Amer. Math. Soc., to appear.

11. PERVIN, W., Quasi-Uniformization of Topological Spaces, Math. Ann. 147 (1962), 316-317.

12. NAIMPALLY, S., Function Spaces of Quasi-Uniform Spaces, Indag. Math., $\underline{68}$ (1965), 768-771. 


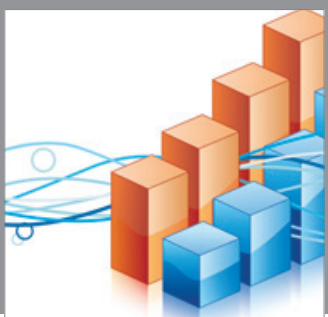

Advances in

Operations Research

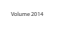

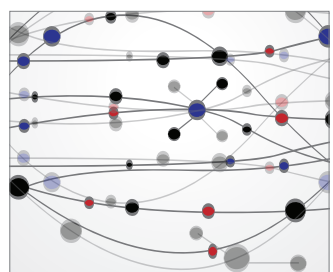

\section{The Scientific} World Journal
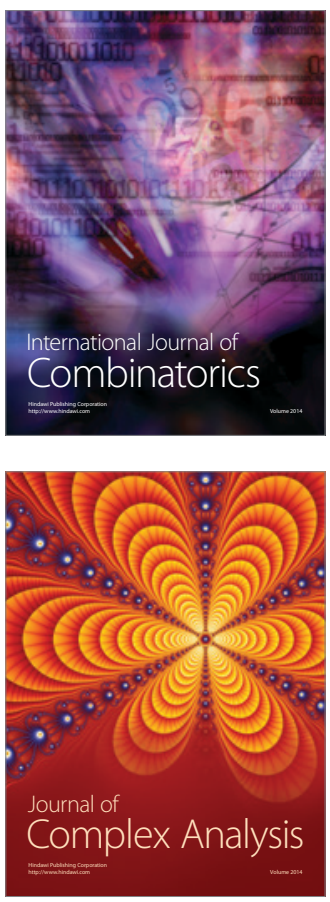

International Journal of

Mathematics and

Mathematical

Sciences
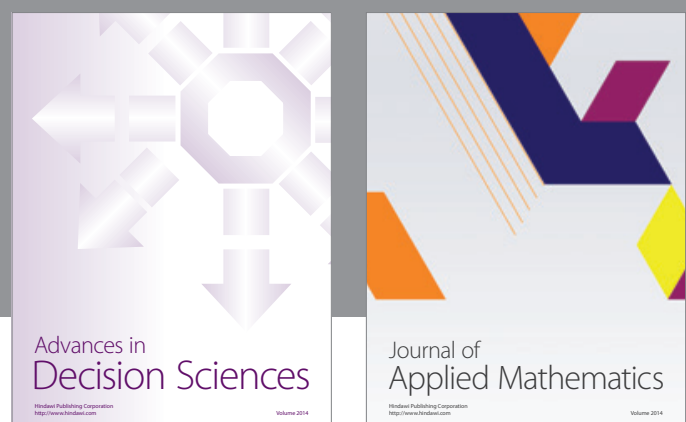

Journal of

Applied Mathematics
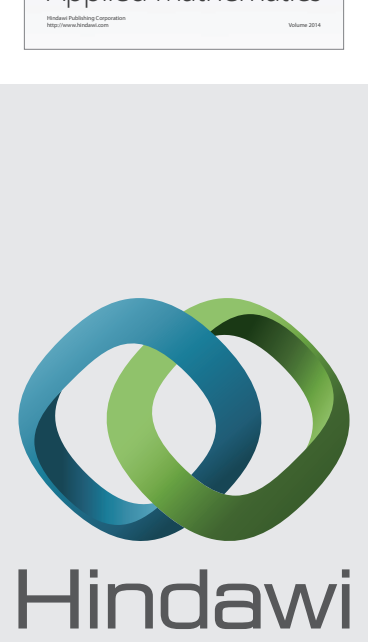

Submit your manuscripts at http://www.hindawi.com
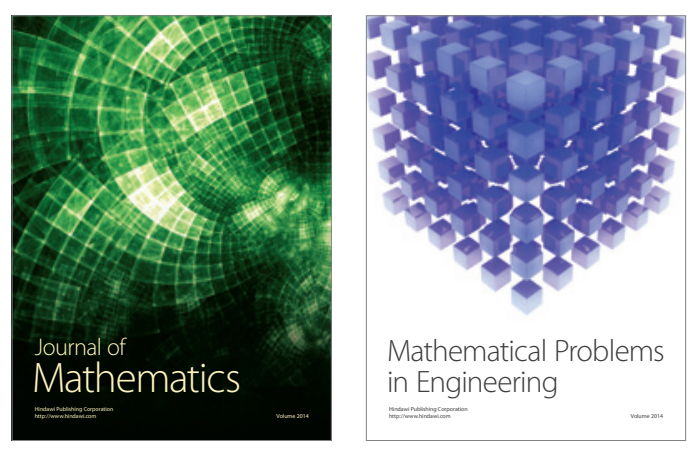

Mathematical Problems in Engineering
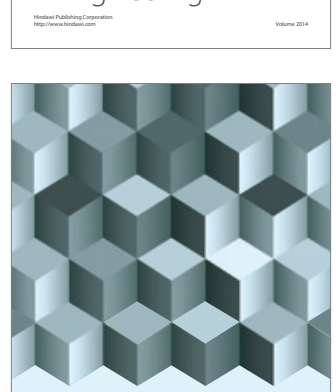

Journal of

Function Spaces
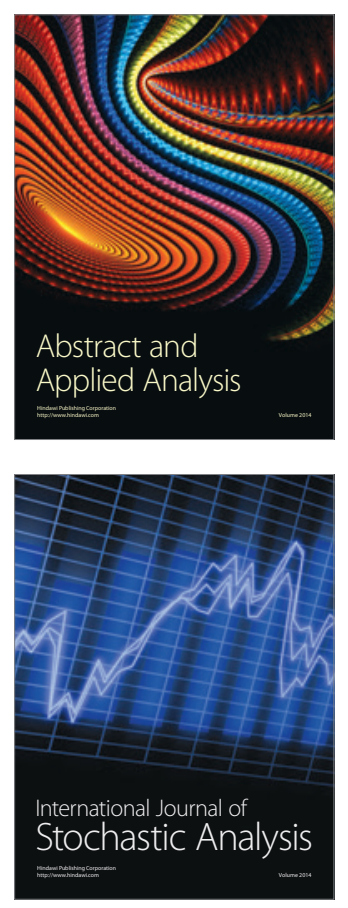

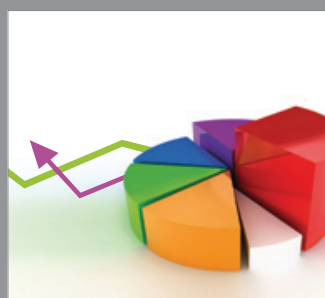

ournal of

Probability and Statistics

Promensencen
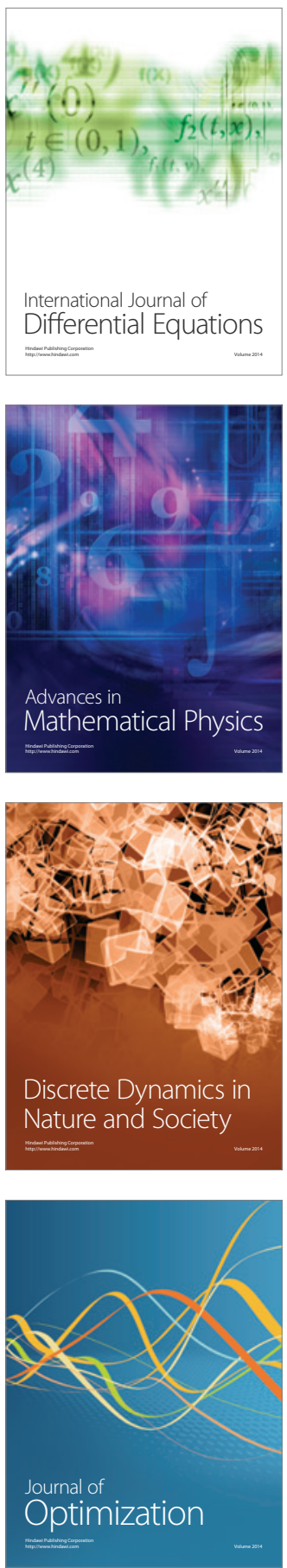